Article

\title{
Burden of Acute Respiratory Infections Among Under-Five Children in Relation to Household Wealth and Socioeconomic Status in Bangladesh
}

\author{
Sanni Yaya $*,+(\mathbb{D}$ and Ghose Bishwajit *,+ \\ Faculty of Social Sciences, School of International Development and Global Studies, University of Ottawa, \\ Ottawa, ON K1N 6N5, Canada \\ * Correspondence: sanni.yaya@uottawa.ca (S.Y.); brammaputram@gmail.com (G.B.); \\ Tel.: +1-613-562-5800 (S.Y.); +1-613-262-4852 (G.B.) \\ + These authors contributed equally to this work.
}

Received: 7 December 2018; Accepted: 8 February 2019; Published: 12 February 2019

check for updates

\begin{abstract}
Acute respiratory infections (ARIs), as a group of diseases and symptoms, are a leading cause of morbidity and mortality among under-five children in tropical countries like Bangladesh. Currently, no clear evidence has been published on the prevalence and socioeconomic correlates of ARIs in Bangladesh. In this regard, we carried out this study with the aim of assessing the prevalence and the socioeconomic predictors of ARIs among children aged 0-59 months, with a special focus on socioeconomic status and wealth-related indicators. Cross-sectional data on 32,998 mother-child (singleton) pairs were collected from six rounds of Bangladesh Demographic and Health Surveys (BDHS 1997-2014). The outcome variable were presence of the common symptoms of ARIs, fever and dyspnea, during the previous two weeks, which were measured based on mothers' reports about the symptoms of these conditions. Explanatory variables included maternal demographic and socioeconomic factors such as age, education, occupation, wealth quintile, and child's age and sex. The prevalence and predictors of ARIs were measured using descriptive and multivariate regression methods. The prevalence of both fever (31.00\% in 1997 vs. 36.76\% in 2014) and dyspnea (39.27\% in 1997 vs. $43.27 \%$ in 2014) has increased gradually since 1997, and tended to be higher in households in the lower wealth quintiles. Multivariable analysis revealed that higher maternal educational status, access to improved water and sanitation facilities, and living in households in higher wealth quintiles had protective effects against both fever and dyspnea. Findings suggested a significantly negative association between lacking access to improved water and sanitation and use of biomass fuel with ARI symptoms. However, no sex difference was observed in these associations. Based on the findings, childhood ARI prevention strategies should address the risk factors stemming from parental socioeconomic marginalisation, household water and sanitation poverty, and use of unclean fuel.
\end{abstract}

Keywords: acute respiratory infections; fever; dyspnea; Bangladesh Demographic and Health Survey; socioeconomic status; household wealth

\section{Introduction}

With the sixth largest population on earth, Bangladesh has the eighth largest population in poverty [1], and the highest percentage of the population below the national poverty line among all South Asian countries [2]. Since its independence in 1971, the country has made remarkable strides in reducing extreme poverty and promoting key population health indicators such as maternal and child mortality, and providing access to basic public health services [3,4]. Bangladesh continues to face overwhelming challenges in meeting the basic healthcare needs of the population. The situation is particularly challenging among vulnerable groups of the population, such as women and children [5-8]. 
According to the United Nations International Children's Emergency Fund (UNICEF), about half of all Bangladeshi children ( $\sim 33$ million) are living in poverty, with one-quarter being deprived of basic needs including food, education, health, and sanitation [9]. The impact of economic poverty on child health is further aggravated by chronic water and sanitation insecurity among the disadvantaged populations [10-13]. The World Health Organization (WHO) has estimated that $60 \%$ of the population in Bangladesh lacks access to improved sources of water and sanitation [14]. The persisting water and sanitation poverty represent a serious challenge for promoting child health in Bangladesh [10,15-17].

The entrenched economic, water, and sanitation poverty is reflected in the high burden of infectious diseases of poverty such as acute respiratory infections (ARIs), which represent the largest share of disease burden in Bangladesh [18]. ARIs, as a group of diseases, are classified as upper or lower respiratory tract infections whose negative effects can include infection, inflammation, and reduced lung function [19-21]. ARIs are the most common causes of morbidity and mortality among under-five children (except for neonates) [19]. Evidence from studies in India and sub-Saharan African countries suggests that poverty influences child health and disease outcomes, including ARIs, through various direct and indirect pathways [22-25]. For many poor households in Bangladesh, especially in rural areas, lack of electricity and clean fuel are common concerns that are circumvented by the use of biomass fuel such as coal, solid domestic waste, dried leaves and wood, and often kerosene [26-28]. Biomass fuel burning is known to be a major cause of indoor air pollution because of the organic material emissions, such as nitrogen oxides, carbon monoxide, and hazardous air pollutants (HAPs), which thereby contribute to the higher burden of ARIs [27,29,30]. However, no research evidence has been published on these associations among under-five children in Bangladesh. To address this research gap, in the present study, we analysed several nationally-representative datasets from the Bangladesh Demographic and Health Survey. The main objective was to measure the prevalence of ARIs among under-five children during the last two decades. We examined sociodemographic patterns in the prevalence of ARIs, with special focus on household wealth status and lack of improved water and sanitation facilities.

\section{Methods}

\subsection{Data Sources}

The study is based on cross-sectional data from six rounds of Bangladesh Demographic and Health Survey conducted between 1997 and 2014. The surveys are conducted by the National Institute of Population Research and Training of Bangladesh and Mitra and Associates with financial support provided by the U.S. Agency for International Development (USAID) and technical assistance by ICF (Inner City Fund) International of Calverton (NY), USA. The surveys are nationally representative and employ multistage sampling strategies for data collection. In general, the sampling frame consists of clusters (known as primary sampling units (PSUs) or enumeration areas) covering both urban and rural areas across the country. Afterward, the PSUs are stratified into homogenous subgroups (Strata) to ensure the representativeness of the sample. Households are then randomly selected from each stratum to interview eligible men and women. Further details of the sampling strategy, response rates, and questionnaires were published previously [31,32].

\subsection{Measures}

The outcome variable was the occurrence of ARI symptoms during the previous two weeks: (1) fever and (2) dyspnea for the youngest child in the household. This was assessed by asking the mothers about: (1) child having a fever during the last two weeks, and (2) child suffering from short, rapid breaths during last two weeks $[22,25,30]$. The answers were categorized as yes or no (no or do not know). In this paper, the term ARI refers to symptoms of ARIs.

Depending on the availability of the datasets, as well their theoretical relationship known from the literature $[22,23,25,33]$, the following maternal demographic and socioeconomic (e.g., education and 
occupation) [34] and child-level variables were selected as the potential predictors of ARI: maternal age (15-19, 20-24, 25-29, or 30-34); residency (urban/ rural); region (Barisal Chittagong, Dhaka, Khulna, Rajshahi, Rangpur, or Sylhet); literacy level (no education, primary, secondary, higher); religion (Islam, other); sanitation (unimproved, improved); water (unimproved, improved); cooking fuel (unclean, clean); wealth quintile (poorest, poorer middle, richer, richest); employment (unemployed, employed); parity $(2,4,5)$; age of child (0-11, 12-23, 24-35, 36-47, 48-59); and child's sex (male/ female). Cooking fuel was categorized as clean if electricity, biogas, or liquefied petroleum gas were used, and unclean if kerosene or wood were used. Household wealth quintile was calculated based on scores of household possession of durable goods (e.g., television, refrigerator) using principal component analysis. The final scores were categorized into quintiles (poorest: q1, poorer: q2, middle: q3, richer: q4; and richest: Q5) so that higher quintiles represent better wealth status [35].

\subsection{Data Analysis}

Data were analysed with Stata Corp (College Station, Texas) version 14. Datasets were first cleaned, checked for missing values, outliers, multicollinearity issues (provided in the Appendix A, Table A2), and merged in order to perform pooled analysis. Then, the dataset was prepared for cluster analysis by accounting for sampling design, sampling strata, and weight. From this point on, all the analyses were performed using the survey command. Sample characteristics are described by percentages with $95 \%$ confidence intervals (CIs). The prevalence of fever and dyspnea stratified by age groups and wealth quintile are presented as bar charts for all six rounds. As the outcome variables were dichotomous, binary logistic regression methods were applied to assess the relationship between fever and dyspnoea. Regression models for both of the outcome measures were stratified by child's sex, and are presented as odds ratios and $95 \%$ CIs. All tests are two-tailed and were considered significant at alpha value of $5 \%$.

For ethical clearance, all participants provided informed consent prior to participating in the survey. Further approval was not necessary for this study as the data were secondary and are available in public domain in anonymized form.

\section{Results}

\subsection{Sample Characteristics}

Basic sociodemographic characteristics of the sample population are summarised in Table A1 (available in the Appendix A). The combined prevalence of fever among boys and girls was 51.6\% $(95 \% \mathrm{CI}=50.5-52.6)$ and $48.4 \%(95 \% \mathrm{CI}=47.4-49.5)$ respectively- and that of dyspnoea was $52.7 \%(95 \%$ $\mathrm{CI}=51.0-54.3)$ and $47.3 \%(95 \% \mathrm{CI}=45.7-49.0)$-respectively. The sex differences were not statistically significant. In 2014-the overall prevalence of fever was $36.76 \%(95 \% \mathrm{CI}=34.9-38.1)$ and that of and dyspnoea was $43.27 \%$ (95\% CI = 42.4-44.6) —both being higher than their 1997 levels: fever $31.0 \%$ (95\% $\mathrm{CI}=29.9-32.4)$ and dyspnoea at $39.27 \%(95 \% \mathrm{CI}=38.2-41.1)$.

Figure 1 illustrates the prevalence of fever among under-five children for all the survey years. Since 1997, the prevalence declined, albeit slowly, among the younger age groups aged 0-11 and 12-23 months, but increased for the older children aged 36-47 and 48-59 months.

Figure 2 illustrates the prevalence of dyspnoea among under-five children for all the survey years. Since 1997, the prevalence declined only among the youngest groups aged 0-11 months and increased for the older children. During 1997-2014, the largest increase was observed in the highest age group of 48-59 months.

As shown in Figure 3, in 1997 the prevalence of fever was relatively higher in the lower wealth quintiles (poorer and poorest) than the higher quintiles (richer and richest). The pattern was somewhat similar for most of the surveys; the combined prevalence in the higher wealth quintiles was constantly lower than in the lower quintiles. This difference was statistically significant $(p<0.05)$. 

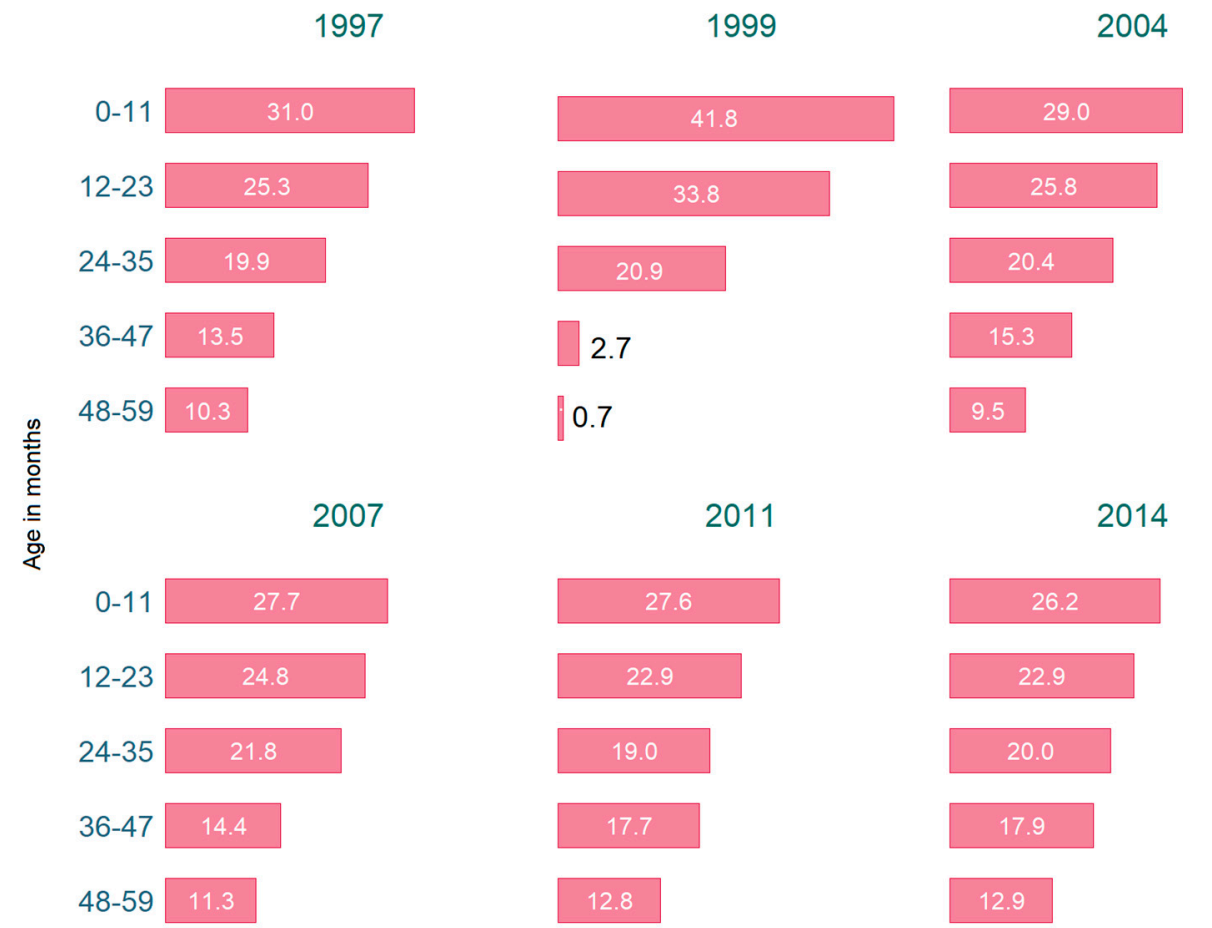

2014

Prevalence of fever by age groups (\%)

Figure 1. Prevalence of fever among under-five children in Bangladesh 1997-2014.
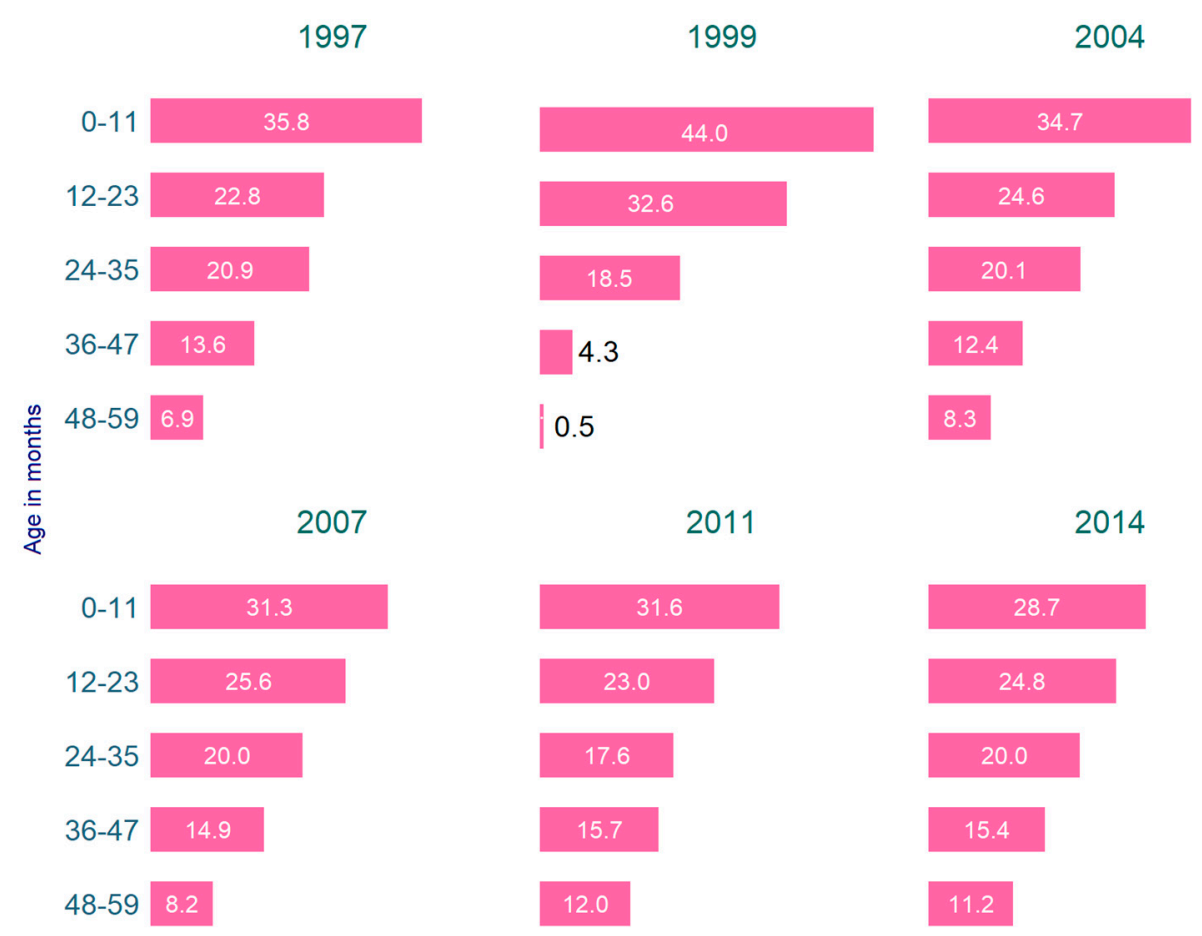

Prevalence of dyspnea by age groups (\%)

Figure 2. Prevalence of dyspnoea among under-five children in Bangladesh 1997-2014. 


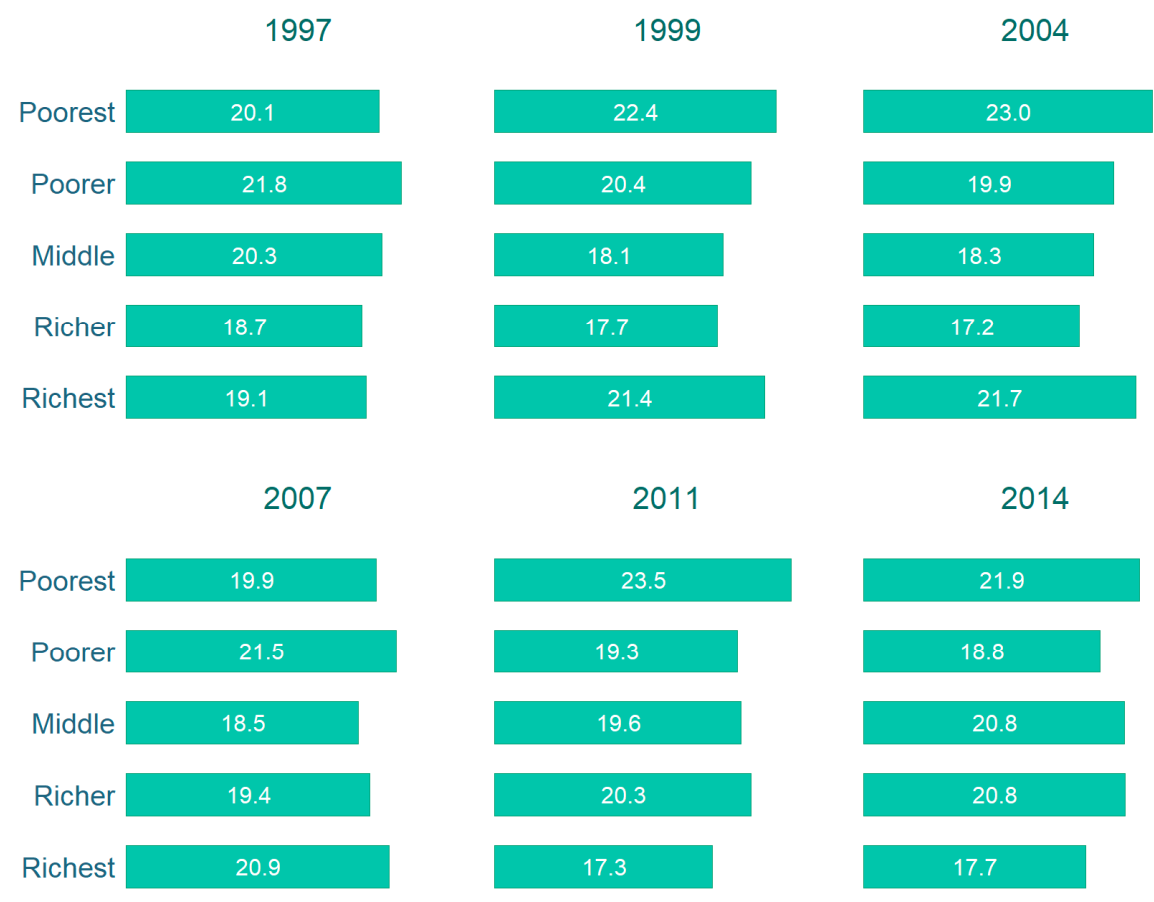

Prevalence of fever by wealth quintile (\%)

Figure 3. Prevalence of fever among under-five children by household wealth quintile in Bangladesh 1997-2014.

Similar to our findings on fever, Figure 4 shows that the prevalence of dyspnoea was generally higher in the lower wealth quintiles (poorer and poorest) than in the higher quintiles (richer and richest) for all survey years except for 1997. This difference was also statistically significant $(p<0.05)$.

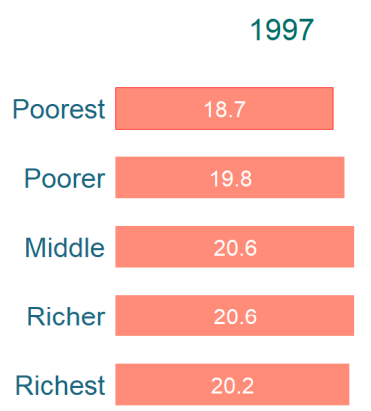

2007

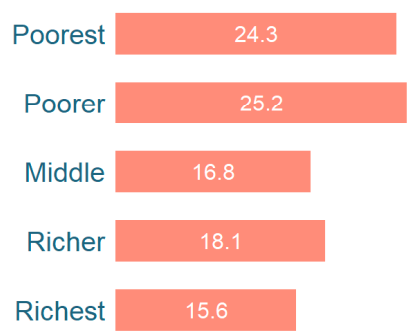

1999

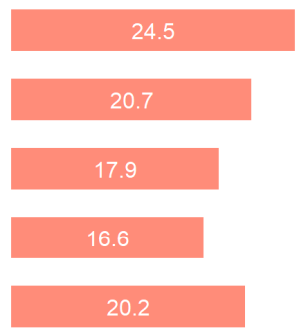

2011

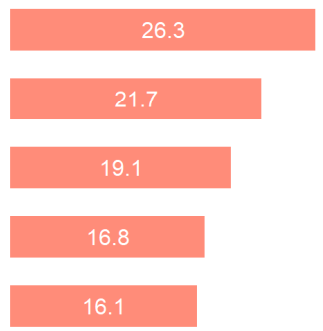

2004

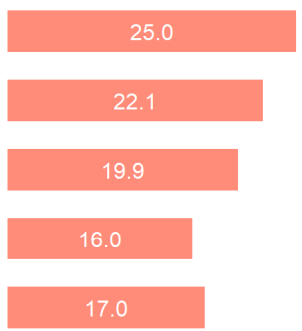

2014

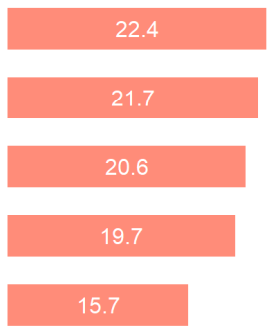

Prevalence of dyspnea by wealth quintile (\%)

Figure 4. Prevalence of dyspnoea among under-five children by household wealth quintile in Bangladesh 1997-2014. 


\subsection{Multivariable Analysis}

The odds ratios (ORs) of the associations between the predictor variables with fever, dyspnea, and both fever and dyspnoea are presented in Tables 1-3, respectively. Mother's age and religion did not show any significant association with fever. However, place of residency, region (for Khulna and Rajshahi only), education, and employment appeared to be significant predictors of fever and dyspnoea. The odds of fever were lower in Khulna (OR $=0.850,95 \% \mathrm{CI}=0.723-0.999)$ but higher in the Rajshahi district (OR $=1.163,95 \% \mathrm{CI}=1.001-1.352)$ (Table 1), whereas that of dyspnoea was lower in Rajshahi (OR $=0.770,95 \% \mathrm{CI}=0.607,0.976)$ (Table 2). The odds of fever (OR $=0.809,95 \%$ $\mathrm{CI}=0.679-0.963)$ and dyspnoea $(\mathrm{OR}=0.584,95 \% \mathrm{CI}=0.384-0.890)$ were significantly lower when mothers had higher education levels. Children of unemployed mothers had higher odds of fever $(\mathrm{OR}=1.117,95 \% \mathrm{CI}=1.016-1.227)$, but not of dyspnoea. However, most of these associations lost their significance after stratifying by child's sex. The odds of fever were lower for households in the higher wealth quintiles (e.g., $\mathrm{OR}=0.824,95 \% \mathrm{CI}=0.701-0.968$ for the highest wealth quintile), and with access to improved water $(\mathrm{OR}=0.856,95 \% \mathrm{CI}=0.659-0.988)$ and sanitation ( $\mathrm{OR}=0.738,95 \% \mathrm{CI}=0.652-0.932)$. Similar findings were found for dyspnoea and for the combined association of fever and dyspnea (Table 3). Using clean fuel (OR $=0.648,95 \% \mathrm{CI}=0.464-0.903)$ and having access to improved sanitation $(0.64495 \% \mathrm{CI}=0.466-0.890)$ were associated lowers odds of having ARI symptoms. Compared with the poorest households, children in the richer $(\mathrm{OR}=0.581,95 \% \mathrm{CI}=0.352-0.959)$ and richest $(0.592$, $95 \% \mathrm{CI}=0.357-0.980$ ) households had lower odds of having ARI symptoms.

Table 1. Predictors of fever among under-five children in Bangladesh, Bangladesh Demographic and Health Survey 1997-2014.

\begin{tabular}{llll}
\hline & Overall & Male & Female \\
\hline Age (15-19) & 1 & 1 & 1 \\
\hline $20-24$ & 1.041 & 0.932 & 1.166 \\
$25-29$ & $(0.923,1.175)$ & $(0.786,1.105)$ & $(0.981,1.386)$ \\
& 0.945 & 0.886 & 1.019 \\
30+ & $(0.831,1.075)$ & $(0.738,1.064)$ & $(0.849,1.223)$ \\
& 0.987 & 0.910 & 1.076 \\
\hline Residency (Urban) & $(0.862,1.131)$ & $(0.750,1.104)$ & $(0.889,1.303)$ \\
\hline Rural & 1 & 1 & 1 \\
& $0.889 *$ & 0.877 & 0.899 \\
Region (Barisal) & $(0.807,0.980)$ & $(0.765,1.006)$ & $(0.782,1.032)$ \\
\hline Chittagong & 1 & 1 & 1 \\
Dhaka & 1.133 & $1.458^{* * *}$ & 0.895 \\
Khulna & $(0.980,1.310)$ & $(1.186,1.794)$ & $(0.728,1.100)$ \\
Rajshahi & 0.965 & 1.161 & 0.813 \\
Rangpur & $(0.831,1.120)$ & $(0.939,1.436)$ & $(0.658,1.004)$ \\
Sylhet & $0.850^{*}$ & 0.958 & $0.765 *$ \\
& $(0.723,0.999)$ & $(0.759,1.208)$ & $(0.610,0.960)$ \\
\hline & $1.163^{*}$ & $1.391 * *$ & 0.994 \\
& $(1.001,1.352)$ & $(1.123,1.722)$ & $(0.803,1.230)$ \\
& 1.011 & $1.332^{*}$ & $0.766 *$ \\
& $(0.862,1.185)$ & $(1.063,1.668)$ & $(0.609,0.963)$ \\
& 1.133 & $1.512^{* *}$ & 0.850 \\
& $(0.950,1.352)$ & $(1.179,1.938)$ & $(0.660,1.095)$ \\
\hline
\end{tabular}


Table 1. Cont.

\begin{tabular}{|c|c|c|c|}
\hline & Overall & Male & Female \\
\hline Education (None) & 1 & 1 & 1 \\
\hline Primary & $\begin{array}{l}1.031 \\
(0.922,1.153)\end{array}$ & $\begin{array}{l}0.999 \\
(0.855,1.167)\end{array}$ & $\begin{array}{l}1.078 \\
(0.917,1.269)\end{array}$ \\
\hline Secondary & $\begin{array}{l}1.013 \\
(0.902,1.138)\end{array}$ & $\begin{array}{l}0.907 \\
(0.772,1.066)\end{array}$ & $\begin{array}{l}1.144 \\
(0.967,1.353)\end{array}$ \\
\hline Higher & $\begin{array}{l}0.809 * \\
(0.679,0.963)\end{array}$ & $\begin{array}{l}0.767 * \\
(0.601,0.979)\end{array}$ & $\begin{array}{l}0.853 \\
(0.664,1.096)\end{array}$ \\
\hline Religion (Islam) & 1 & 1 & 1 \\
\hline Others & $\begin{array}{l}0.894 \\
(0.689,1.216)\end{array}$ & $\begin{array}{l}0.773 \\
(0.552,1.121)\end{array}$ & $\begin{array}{l}0.960 \\
(0.781,1.181)\end{array}$ \\
\hline $\begin{array}{l}\text { Sanitation (Unimproved) } \\
\text { Improved }\end{array}$ & $\begin{array}{l}1 \\
0.738^{* *} \\
(0.652,0.932)\end{array}$ & $\begin{array}{l}1 \\
0.977 \\
(0.854,1.116)\end{array}$ & $\begin{array}{l}1 \\
0.900 \\
(0.784,1.032)\end{array}$ \\
\hline Water (Unimproved) & 1 & 1 & 1 \\
\hline Improved & $\begin{array}{l}0.856 * * \\
(0.659,0.988)\end{array}$ & $\begin{array}{l}1.035 \\
(0.975,1.565)\end{array}$ & $\begin{array}{l}0.982 \\
(0.702,1.639)\end{array}$ \\
\hline Fuel (Unclean) & 1 & 1 & 1 \\
\hline Clean & $\begin{array}{l}0.830 * * \\
(0.593,0.991)\end{array}$ & $\begin{array}{l}0.817 \\
(0.631,1.150)\end{array}$ & $\begin{array}{l}0.938 \\
(0.749,1.176)\end{array}$ \\
\hline Employed (Yes) & 1 & 1 & 1 \\
\hline No & $\begin{array}{l}1.117 * \\
(1.016,1.227)\end{array}$ & $\begin{array}{l}1.231 * * \\
(1.078,1.405)\end{array}$ & $\begin{array}{l}1.002 \\
(0.874,1.148)\end{array}$ \\
\hline Wealth (Poorest) & 1 & 1 & 1 \\
\hline Poorer & $\begin{array}{l}0.947 \\
(0.835,1.073)\end{array}$ & $\begin{array}{l}0.869 \\
(0.731,1.034)\end{array}$ & $\begin{array}{l}1.035 \\
(0.863,1.241)\end{array}$ \\
\hline Middle & $\begin{array}{l}0.940 \\
(0.825,1.069)\end{array}$ & $\begin{array}{l}0.855 \\
(0.713,1.025)\end{array}$ & $\begin{array}{l}1.019 \\
(0.846,1.227)\end{array}$ \\
\hline Richer & $\begin{array}{l}0.869 * \\
(0.757,0.998)\end{array}$ & $\begin{array}{l}0.831 \\
(0.685,1.009)\end{array}$ & $\begin{array}{l}0.913 \\
(0.749,1.114)\end{array}$ \\
\hline Richest & $\begin{array}{l}0.824 * \\
(0.701,0.968)\end{array}$ & $\begin{array}{l}0.748 * \\
(0.593,0.942)\end{array}$ & $\begin{array}{l}0.913 \\
(0.726,1.147)\end{array}$ \\
\hline Child's Age (0-11 months) & 1 & 1 & 1 \\
\hline $12-23$ & $\begin{array}{l}0.973 \\
(0.870,1.088)\end{array}$ & $\begin{array}{l}0.938 \\
(0.801,1.099)\end{array}$ & $\begin{array}{l}1.002 \\
(0.855,1.173)\end{array}$ \\
\hline $24-35$ & $\begin{array}{l}0.803^{* * *} \\
(0.715,0.902)\end{array}$ & $\begin{array}{l}0.769 * * \\
(0.654,0.905)\end{array}$ & $\begin{array}{l}0.828 * \\
(0.701,0.979)\end{array}$ \\
\hline $36-47$ & $\begin{array}{l}0.772 * * * \\
(0.682,0.873)\end{array}$ & $\begin{array}{l}0.713 * * * \\
(0.599,0.848)\end{array}$ & $\begin{array}{l}0.838 \text { * } \\
(0.702,1.000)\end{array}$ \\
\hline $48-59$ & $\begin{array}{l}0.631 * * * \\
(0.550,0.725)\end{array}$ & $\begin{array}{l}0.645^{* * *} \\
(0.533,0.780)\end{array}$ & $\begin{array}{l}0.607 * * * \\
(0.497,0.743)\end{array}$ \\
\hline \multicolumn{4}{|l|}{ Sex (Male) } \\
\hline Female & $\begin{array}{l}0.911 \\
(0.705,1.143)\end{array}$ & NA & NA \\
\hline Nagalekerke- $R^{2}$ & 0.341 & 0.419 & 0.368 \\
\hline
\end{tabular}

Note: All models are adjusted for year of survey. Figures represent odds ratios with $95 \%$ confidence intervals, reference categories in () brackets. ${ }^{*} p<0.05,{ }^{* *} p<0.01,{ }^{* * *} p<0.001$. 
Table 2. Predictors of dyspnea among under-five children in Bangladesh. Bangladesh Demographic and Health Survey 1997-2014.

\begin{tabular}{|c|c|c|c|}
\hline & Overall & Male & Female \\
\hline Age (15-19) & 1 & 1 & 1 \\
\hline $20-24$ & $\begin{array}{l}0.802 \\
(0.666,1.065)\end{array}$ & $\begin{array}{l}0.828 \\
(0.641,1.068)\end{array}$ & $\begin{array}{l}0.774 \\
(0.587,1.019)\end{array}$ \\
\hline $25-29$ & $\begin{array}{l}0.888 \\
(0.643,1.464)\end{array}$ & $\begin{array}{l}0.679 * * \\
(0.513,0.898)\end{array}$ & $\begin{array}{l}0.934 \\
(0.694,1.257)\end{array}$ \\
\hline $30+$ & $\begin{array}{l}0.921 \\
(0.745,1.139)\end{array}$ & $\begin{array}{l}0.940 \\
(0.698,1.265)\end{array}$ & $\begin{array}{l}0.914 \\
(0.671,1.243)\end{array}$ \\
\hline Residency (Urban) & 1 & 1 & 1 \\
\hline Rural & $\begin{array}{l}0.900 \\
(0.771,1.051)\end{array}$ & $\begin{array}{l}1.020 \\
(0.823,1.263)\end{array}$ & $\begin{array}{l}0.777 * \\
(0.619,0.975)\end{array}$ \\
\hline Region (Barisal) & 1 & 1 & 1 \\
\hline Chittagong & $\begin{array}{l}1.020 \\
(0.812,1.281)\end{array}$ & $\begin{array}{l}1.131 \\
(0.822,1.557)\end{array}$ & $\begin{array}{l}0.923 \\
(0.664,1.282)\end{array}$ \\
\hline Dhaka & $\begin{array}{l}0.983 \\
(0.774,1.247)\end{array}$ & $\begin{array}{l}1.150 \\
(0.818,1.616)\end{array}$ & $\begin{array}{l}0.839 \\
(0.598,1.176)\end{array}$ \\
\hline Khulna & $\begin{array}{l}0.850 \\
(0.658,1.098)\end{array}$ & $\begin{array}{l}0.941 \\
(0.656,1.351)\end{array}$ & $\begin{array}{l}0.757 \\
(0.525,1.092)\end{array}$ \\
\hline Rajshahi & $\begin{array}{l}0.770 * \\
(0.607,0.976)\end{array}$ & $\begin{array}{l}0.776 \\
(0.554,1.086)\end{array}$ & $\begin{array}{l}0.760 \\
(0.542,1.067)\end{array}$ \\
\hline Rangpur & $\begin{array}{l}0.789 \\
(0.609,1.022)\end{array}$ & $\begin{array}{l}0.887 \\
(0.618,1.273)\end{array}$ & $\begin{array}{l}0.688 \\
(0.471,1.003)\end{array}$ \\
\hline Sylhet & $\begin{array}{l}0.950 \\
(0.710,1.272)\end{array}$ & $\begin{array}{l}1.154 \\
(0.775,1.720)\end{array}$ & $\begin{array}{l}0.763 \\
(0.492,1.183)\end{array}$ \\
\hline Education (None) & 1 & 1 & 1 \\
\hline Primary & $\begin{array}{l}1.051 \\
(0.881,1.254)\end{array}$ & $\begin{array}{l}1.214 \\
(0.952,1.547)\end{array}$ & $\begin{array}{l}0.899 \\
(0.692,1.167)\end{array}$ \\
\hline Secondary & $\begin{array}{l}0.823 * \\
(0.683,0.992)\end{array}$ & $\begin{array}{l}0.916 \\
(0.707,1.187)\end{array}$ & $\begin{array}{l}0.724 * \\
(0.551,0.951)\end{array}$ \\
\hline Higher & $\begin{array}{l}0.584 \text { * } \\
(0.384,0.890)\end{array}$ & $\begin{array}{l}0.961 \\
(0.639,1.445)\end{array}$ & $\begin{array}{l}0.758 \\
(0.567,1.013)\end{array}$ \\
\hline Religion (Islam) & 1 & 1 & 1 \\
\hline Others & $\begin{array}{l}0.873 \\
(0.690,1.105)\end{array}$ & $\begin{array}{l}0.758 \\
(0.547,1.052)\end{array}$ & $\begin{array}{l}0.993 \\
(0.704,1.401)\end{array}$ \\
\hline Sanitation (Unimproved) & 1 & 1 & 1 \\
\hline Improved & $\begin{array}{l}0.724^{* *} \\
(0.595,8.073)\end{array}$ & $\begin{array}{l}1.009 \\
(0.817,1.244)\end{array}$ & $\begin{array}{l}0.836 \\
(0.672,1.041)\end{array}$ \\
\hline Water (Unimproved) & 1 & 1 & 1 \\
\hline Improved & $\begin{array}{l}0.820 * \\
(0.672,0.949)\end{array}$ & $\begin{array}{l}0.941 \\
(0.714,1.518)\end{array}$ & $\begin{array}{l}1.014 \\
(0.667,1.544)\end{array}$ \\
\hline Fuel (Unclean) & 1 & 1 & 1 \\
\hline Clean & $\begin{array}{l}0.682 * * \\
(0.520,0.894)\end{array}$ & $\begin{array}{l}0.635 * \\
(0.433,0.930)\end{array}$ & $\begin{array}{l}0.717 \\
(0.485,1.060)\end{array}$ \\
\hline Employed (Yes) & 1 & 1 & 1 \\
\hline No & $\begin{array}{l}0.987 \\
(0.848,1.149)\end{array}$ & $\begin{array}{l}0.953 \\
(0.772,1.177)\end{array}$ & $\begin{array}{l}1.021 \\
(0.818,1.275)\end{array}$ \\
\hline
\end{tabular}


Table 2. Cont.

\begin{tabular}{|c|c|c|c|}
\hline & Overall & Male & Female \\
\hline Wealth (Poorest) & 1 & 1 & 1 \\
\hline Poorer & $\begin{array}{l}1.162 \\
(0.953,1.416)\end{array}$ & $\begin{array}{l}1.067 \\
(0.813,1.400)\end{array}$ & $\begin{array}{l}1.279 \\
(0.954,1.715)\end{array}$ \\
\hline Middle & $\begin{array}{l}1.030 \\
(0.838,1.266)\end{array}$ & $\begin{array}{l}0.882 \\
(0.663,1.173)\end{array}$ & $\begin{array}{l}1.210 \\
(0.892,1.640)\end{array}$ \\
\hline Richer & $\begin{array}{l}0.849 * \\
(0.681,0.960)\end{array}$ & $\begin{array}{l}0.794 \\
(0.583,1.081)\end{array}$ & $\begin{array}{l}0.915 \\
(0.662,1.265)\end{array}$ \\
\hline Richest & $\begin{array}{l}0.784 \text { * } \\
(0.605,0.916)\end{array}$ & $\begin{array}{l}0.740 \\
(0.514,1.067)\end{array}$ & $\begin{array}{l}0.842 \\
(0.579,1.224)\end{array}$ \\
\hline Child's Age (0-11 months) & 1 & 1 & 1 \\
\hline $12-23$ & $\begin{array}{l}0.873 \\
(0.734,1.038)\end{array}$ & $\begin{array}{l}0.760 * \\
(0.596,0.968)\end{array}$ & $\begin{array}{l}1.019 \\
(0.793,1.308)\end{array}$ \\
\hline $24-35$ & $\begin{array}{l}0.812 \text { * } \\
(0.676,0.976)\end{array}$ & $\begin{array}{l}0.704^{* *} \\
(0.547,0.907)\end{array}$ & $\begin{array}{l}0.951 \\
(0.725,1.248)\end{array}$ \\
\hline $36-47$ & $\begin{array}{l}0.661 \text { *** } \\
(0.540,0.809)\end{array}$ & $\begin{array}{l}0.521 \text { *** } \\
(0.394,0.690)\end{array}$ & $\begin{array}{l}0.863 \\
(0.641,1.160)\end{array}$ \\
\hline $48-59$ & $\begin{array}{l}0.639 * * * \\
(0.508,0.804)\end{array}$ & $\begin{array}{l}0.600 * * \\
(0.439,0.821)\end{array}$ & $\begin{array}{l}0.693^{*} \\
(0.492,0.978)\end{array}$ \\
\hline \multicolumn{4}{|l|}{ Sex (Male) } \\
\hline Female & $\begin{array}{l}0.946 \\
(0.835,1.073)\end{array}$ & NA & NA \\
\hline Nagalekerke- $R^{2}$ & 0.613 & 0.441 & 0.468 \\
\hline
\end{tabular}

Note: All models are adjusted for year of survey. Figures represent odds ratios with $95 \%$ confidence intervals; reference categories in () brackets. ${ }^{*} p<0.05,{ }^{* *} p<0.01, * * * p<0.001$. Goodness of fit of the regression models was assessed by Nagalekerke- $R^{2}$ values that indicated moderate to good predictive capacity for all the models.

Table 3. Predictors of fever and dyspnea among under-five children in Bangladesh. Bangladesh Demographic and Health Survey 1997-2014.

\begin{tabular}{llll}
\hline & Overall & Male & Female \\
\hline Age (15-19) & 1 & 1 & 1 \\
\hline 20-24 & 1.035 & 0.997 & 1.067 \\
$25-29$ & $(0.802,1.335)$ & $(0.695,1.431)$ & $(0.740,1.537)$ \\
30+ & 1.133 & 0.879 & $1.560^{*}$ \\
& $(0.857,1.498)$ & $(0.597,1.294)$ & $(1.032,2.358)$ \\
\hline Residency (Urban) & 1.321 & 1.037 & $1.711^{*}$ \\
\hline Rural & $(0.979,1.782)$ & $(0.680,1.581)$ & $(1.107,2.644)$ \\
& 1 & 1 & 1 \\
\hline Region (Barisal) & 0.955 & 0.972 & 0.911 \\
\hline Chittagong & $(0.772,1.181)$ & $(0.723,1.307)$ & $(0.666,1.246)$ \\
\hline \multirow{2}{*}{ Dhaka } & 1 & 1 & 1 \\
Khulna & 1.033 & $1.640 *$ & $0.592^{*}$ \\
& $(0.745,1.430)$ & $(1.058,2.541)$ & $(0.357,0.982)$ \\
Rajshahi & 1.083 & $1.719 *$ & 0.664 \\
Rangpur & $(0.770,1.524)$ & $(1.073,2.754)$ & $(0.394,1.118)$ \\
Sylhet & $0.615^{* *}$ & 0.774 & $0.458^{* *}$ \\
& $(0.438,0.862)$ & $(0.493,1.214)$ & $(0.270,0.778)$ \\
\hline & 0.915 & 1.122 & 0.702 \\
& $(0.654,1.280)$ & $(0.720,1.749)$ & $(0.414,1.191)$ \\
& 0.819 & 1.261 & $0.489^{*}$ \\
& $(0.574,1.170)$ & $(0.774,2.054)$ & $(0.284,0.844)$ \\
& $2.185^{* *}$ & $3.306^{* * *}$ & 1.358 \\
& $(1.312,3.638)$ & $(1.670,6.546)$ & $(0.621,2.970)$ \\
\hline
\end{tabular}


Table 3. Cont.

\begin{tabular}{|c|c|c|c|}
\hline & Overall & Male & Female \\
\hline Education (None) & 1 & 1 & 1 \\
\hline Primary & $\begin{array}{l}1.058 \\
(0.822,1.363)\end{array}$ & $\begin{array}{l}0.923 \\
(0.649,1.312)\end{array}$ & $\begin{array}{l}1.270 \\
(0.873,1.847)\end{array}$ \\
\hline Secondary & $\begin{array}{l}1.138 \\
(0.876,1.480)\end{array}$ & $\begin{array}{l}1.028 \\
(0.711,1.484)\end{array}$ & $\begin{array}{l}1.337 \\
(0.911,1.962)\end{array}$ \\
\hline Higher & $\begin{array}{l}0.892 \\
(0.619,1.285)\end{array}$ & $\begin{array}{l}1.282 \\
(0.741,2.220)\end{array}$ & $\begin{array}{l}0.653 \\
(0.392,1.086)\end{array}$ \\
\hline Religion (Islam) & 1 & 1 & 1 \\
\hline Others & $\begin{array}{l}0.792 \\
(0.588,1.067)\end{array}$ & $\begin{array}{l}0.699 \\
(0.466,1.050)\end{array}$ & $\begin{array}{l}0.844 \\
(0.540,1.320)\end{array}$ \\
\hline Sanitation (Unimproved) & 1 & 1 & 1 \\
\hline Improved & $\begin{array}{l}0.644^{* *} \\
(0.466,0.890)\end{array}$ & $\begin{array}{l}0.962 \\
(0.712,1.299)\end{array}$ & $\begin{array}{l}0.807 \\
(0.650,1.003)\end{array}$ \\
\hline Water (Unimproved) & 1 & 1 & 1 \\
\hline Improved & $\begin{array}{l}1.051 \\
(0.699,1.580)\end{array}$ & $\begin{array}{l}1.120 \\
(0.648,1.935)\end{array}$ & $\begin{array}{l}0.998 \\
(0.534,1.863)\end{array}$ \\
\hline Fuel (Unclean) & 1 & 1 & 1 \\
\hline Clean & $\begin{array}{l}0.648 * \\
(0.464,0.903)\end{array}$ & $\begin{array}{l}0.565 * \\
(0.354,0.901)\end{array}$ & $\begin{array}{l}0.716 \\
(0.439,1.167)\end{array}$ \\
\hline Employed (Yes) & 1 & 1 & 1 \\
\hline No & $\begin{array}{l}1.113 \\
(0.899,1.379)\end{array}$ & $\begin{array}{l}1.153 \\
(0.855,1.556)\end{array}$ & $\begin{array}{l}1.050 \\
(0.768,1.435)\end{array}$ \\
\hline Wealth (Poorest) & 1 & 1 & 1 \\
\hline Poorer & $\begin{array}{l}0.983 \\
(0.736,1.312)\end{array}$ & $\begin{array}{l}0.937 \\
(0.619,1.418)\end{array}$ & $\begin{array}{l}0.990 \\
(0.656,1.494)\end{array}$ \\
\hline Middle & $\begin{array}{l}1.130 \\
(0.832,1.537)\end{array}$ & $\begin{array}{l}0.738 \\
(0.487,1.119)\end{array}$ & $\begin{array}{l}1.127 \\
(0.738,2.933)\end{array}$ \\
\hline Richer & $\begin{array}{l}0.581 \text { * } \\
(0.352,0.959)\end{array}$ & $\begin{array}{l}0.695 \\
(0.446,1.081)\end{array}$ & $\begin{array}{l}0.999 \\
(0.643,1.553)\end{array}$ \\
\hline Richest & $\begin{array}{l}0.592 \text { * } \\
(0.357,0.980)\end{array}$ & $\begin{array}{l}0.850 \\
(0.624,1.157)\end{array}$ & $\begin{array}{l}1.169 \\
(0.703,1.946)\end{array}$ \\
\hline Child's Age (0-11 months) & 1 & 1 & 1 \\
\hline $12-23$ & $\begin{array}{l}1.051 \\
(0.821,1.345)\end{array}$ & $\begin{array}{l}0.970 \\
(0.685,1.374)\end{array}$ & $\begin{array}{l}1.111 \\
(0.780,1.583)\end{array}$ \\
\hline $24-35$ & $\begin{array}{l}0.861 \\
(0.669,1.107)\end{array}$ & $\begin{array}{l}0.950 \\
(0.664,1.360)\end{array}$ & $\begin{array}{l}0.739 \\
(0.515,1.062)\end{array}$ \\
\hline $36-47$ & $\begin{array}{l}0.900 \\
(0.682,1.189)\end{array}$ & $\begin{array}{l}0.696 \\
(0.481,1.008)\end{array}$ & $\begin{array}{l}1.228 \\
(0.793,1.902)\end{array}$ \\
\hline $48-59$ & $\begin{array}{l}0.749 \\
(0.552,1.016)\end{array}$ & $\begin{array}{l}0.857 \\
(0.557,1.317)\end{array}$ & $\begin{array}{l}0.629 * \\
(0.404,0.980)\end{array}$ \\
\hline Sex (Male) & 1 & & \\
\hline Female & $\begin{array}{l}0.976 \\
(0.821,1.161)\end{array}$ & NA & NA \\
\hline Nagalekerke- $R^{2}$ & 0.374 & 0.280 & 0.412 \\
\hline
\end{tabular}

Note: All models are adjusted for year of survey. Figures represent odds ratios with $95 \%$ confidence intervals; reference categories in () brackets. ${ }^{*} p<0.05,{ }^{* *} p<0.01,{ }^{* * *} p<0.001$. 


\section{Discussion}

Acute respiratory infections (ARIs) are a leading cause of morbidity and mortality among under-five children in low-income countries like Bangladesh. Evidence suggests that in 1997-2001, ARIs including pneumonia were major contributors to hospitalization (40\%) among under-five children at primary public care facilities in rural Bangladesh [35]. The findings of the present study are in line with the existing evidence that a remarkable proportion of the under-five children in Bangladesh continue to suffer from ARIs. As the findings indicate, the prevalence of fever and dyspnea has increased over the last 15 years, especially among children aged three to five years old. During 1997-2014, the overall prevalence has slowly but steadily risen, despite a net decline in the prevalence among infants ( $<12$ months). Although previous studies reported significant sex differentials in childhood morbidity and mortality in Bangladesh $[34,36]$, the present study suggests that the male-female disparity is not significant for ARIs.

A high ARI burden was reported in several sub-national studies among South Asian children including Bangladesh (72\% in 2001, urban slums of Dhaka) [37] and India (59.1\% in 2013-2014, Puducherry) [38]. ARI-attributed mortality rates were found to be high in Nepal (20-30\%) [39] and Pakistan (20-30\%) [40]; however, corresponding estimates are currently not available for Bangladesh. Existing studies suggest that the health impacts of the high disease burden among South Asian children are further exacerbated by sub-optimal care seeking behavior, especially among the poorest households [41,42]. In light of the past and current findings, ARIs remain a common health issue among under-five children in Bangladesh, which requires urgent attention from public health stakeholders.

Apart from the high prevalence, the findings also highlight the significant demographic and socioeconomic pattern in the occurrence of ARIs. The odds of fever or dyspnea did not differ noticeably across maternal age groups; however, being an older child appeared to be a protective factor against both fever and dyspnea. Maternal community level factors, such as rural residency and certain regional disparities, were observed in the odds of fever. For instance, compared to those based in Barishal district, the odds of fever were lower in Khulna but higher in Rajshahi, whereas the odds of dyspnea were lower in Rajshahi only. However, the association between region and ARIs was not consistent across the districts. Regarding maternal socioeconomic characteristics, having higher education and living in higher wealth quintile households were inversely associated with fever and dyspnea, indicating a protective effect of better maternal socioeconomic status (SES) on child's experience of ARIs.

The beneficial effect of higher SES on child's health outcomes is generally attributed to better living conditions, better nutritional status, and access to healthcare services. Parental material hardship can affect child health and exposure to illness through various pathways. For instance, financial stress is strongly correlated with child undernutrition, poor cognitive development, and weakened immune system, so can increase the vulnerability to infectious diseases. Children in financially well-off families are more likely to enjoy healthy and secure living facilities with greater access to health-promoting conditions in comparison to those from impoverished families [43]. Several other environmental aspects of poverty, such as inability to afford improved water, sanitation, and hygiene facilities (WASH) and clean cooking fuel, are also strong predictors of acute illnesses due to their link with important risk factors such as of fecal contamination of food, drinking water, utensils, and child's toys [44].

This study reported the prevalence of ARIs among under-five children in Bangladesh, and their association with maternal and household socioeconomic indicators using nationally representative samples. Th information generated from this study should increase the awareness about this ever-growing health issue and help policy makers to implement appropriate policy measures. Our findings have the potential to inspire national WASH and poverty reduction policies in an effort to improve child poverty-reduction and health-promotion strategies. Bangladesh is also a highly disaster-prone country and is vulnerable to climate change, which are important risk factors for ARIs [45]. Growing evidence in this area of public health can help with the development of effective planning, coping, and intervention mechanisms. Apart from our important contributions, our study 
has several limitations. First, the symptoms of ARIs were reported by mothers and were not the result of objective measurement. The variables were self-reported, and are hence subject to reporting bias. Second, there were no data available on household hygiene practices, which is a key predictor of infectious diseases among populations of all ages. There were no data on whether the children were suffering from any specific illness; therefore, it is possible that the symptoms of fever and dyspnea resulted from conditions other than ARIs. However, this gap is expected to be overcome, to a great extent, by the inclusion of water and sanitation variables. Lastly, the data were secondary, and thus no causal inferences can be made about the associations.

\section{Conclusions}

Since 1997, there has been a slow but steady increase in the prevalence of ARI symptoms, such as fever and dyspnea, among under-five children in Bangladesh. Apart from the high prevalence, our study provides several important findings regarding the socioeconomic predictors of these conditions that might be of interest for child health- and WASH-related stakeholders in the country. Higher maternal educational status and employment status showed protective effects against suffering from fever. As expected, lack of improved WASH facilities was significantly associated higher odds of ARI. Of particular concern was the higher burden of ARIs in households in the lower wealth quintiles. Given these findings, we suggest that child-health-related sustainable development programs in Bangladesh should try to emphasize maternal socioeconomic status and ensuring better environmental conditions, such as optimum WASH coverage and use of clean fuel, especially among the most marginalised communities. More studies are required to investigate the broader macroeconomic and sociocultural factors that may underlie the constantly high prevalence of ARIs among under-five children.

Author Contributions: Conceptualization, G.B.; methodology, G.B.; software, G.B.; validation, G.B., S.Y.; formal analysis, G.B.; investigation, S.Y.; resources, G.B.; data curation, G.B.; writing-original draft preparation, G.B.; S.Y.; writing—review and editing, G.B.; S.Y.; visualization, G.B.; supervision, G.B.; project administration, G.B., S.Y.

Funding: Self-funded by G.B.

Acknowledgments: Authors acknowledge the generous provision of the data by DHS program.

Conflicts of Interest: The authors declare no conflict of interest.

\section{Abbreviations}

ARIs

Acute Respiratory Infections

BDHS Bangladesh Demographic and Health Survey

WASH Water, sanitation and hygiene facilities 


\section{Appendix A}

Table A1. Sample characteristics BDHS 1997-2014.

\begin{tabular}{|c|c|c|c|c|c|c|c|}
\hline & & \multicolumn{2}{|c|}{ Fever $(N=30,731)$} & \multirow[t]{2}{*}{$p$-Value } & \multicolumn{2}{|c|}{ Dyspnoea $(N=11,354)$} & \multirow[t]{2}{*}{$p$-Value } \\
\hline & & No & Yes & & No & Yes & \\
\hline \multicolumn{8}{|c|}{ Maternal Indicators } \\
\hline \multicolumn{8}{|l|}{ Age } \\
\hline 15-19 & 15.2 & $15.5(14.9-16.2)$ & $16.6(15.9-17.4)$ & \multirow[t]{4}{*}{$<0.001$} & $16.9(15.9-18.0)$ & $18.8(17.6-20.1)$ & \multirow[t]{4}{*}{$<0.001$} \\
\hline $20-24$ & 32.5 & $32.0(31.1-32.8)$ & $33.5(32.5-34.6)$ & & $33.8(32.5-35.2)$ & $33.3(31.7-34.8)$ & \\
\hline $25-29$ & 27.0 & $27.2(26.5-27.9)$ & $25.9(24.9-26.8)$ & & $26.0(24.8-27.3)$ & $24.9(23.4-26.4)$ & \\
\hline $30-34$ & 25.3 & $25.3(24.6-26.1)$ & $24.0(23.1-24.9)$ & & $23.2(22.0-24.4)$ & $23.0(21.8-24.4)$ & \\
\hline \multicolumn{8}{|l|}{ Residency } \\
\hline Urban & 29.2 & $21.2(19.9-22.5)$ & $19.7(18.3-21.2)$ & \multirow[t]{2}{*}{$<0.001$} & $21.1(19.5-22.8)$ & $19.1(17.5-20.8)$ & \multirow[t]{2}{*}{$<0.001$} \\
\hline Rural & 70.8 & $78.8(77.5-80.1)$ & $80.3(78.8-81.7)$ & & $78.9(77.2-80.5)$ & $80.9(79.2-82.5)$ & \\
\hline \multicolumn{8}{|l|}{ Region } \\
\hline Barisal & 11.4 & $6.1(5.3-6.9)$ & $6.3(5.5-7.2)$ & \multirow[t]{7}{*}{$<0.001$} & $6.1(5.2-7.1)$ & $6.6(5.5-7.8)$ & \multirow[t]{7}{*}{$<0.001$} \\
\hline Chittagong & 17.9 & $19.8(18.2-21.5)$ & $23.3(21.4-25.4)$ & & $22.7(20.6-24.9)$ & $24.7(22.4-27.1)$ & \\
\hline Dhaka & 20.6 & $33.4(31.3-35.5)$ & $30.0(27.8-32.4)$ & & $30.8(28.5-33.1)$ & $28.5(26.1-31.1)$ & \\
\hline Khulna & 12.3 & $11.1(10.0-12.3)$ & $8.6(7.6-9.6)$ & & $9.7(8.6-11.0)$ & $9.6(8.3-10.9)$ & \\
\hline Rajshahi & 18.7 & $18.2(16.6-19.9)$ & $19.5(17.7-21.4)$ & & $19.7(17.7-21.8)$ & $18.4(16.4-20.6)$ & \\
\hline Rangpur & 13.2 & $8.5(7.6-9.5)$ & $8.6(7.6-9.7)$ & & $8.1(6.9-9.6)$ & $8.7(7.5-10.0)$ & \\
\hline Sylhet & 6.0 & $3.1(2.6-3.6)$ & $3.7(3.0-4.6)$ & & $2.9(2.3-3.8)$ & $3.6(2.8-4.6)$ & \\
\hline \multicolumn{8}{|l|}{ Literacy Level } \\
\hline No Education & 29.1 & $30.6(29.5-31.8)$ & $30.3(29.1-31.6)$ & \multirow[t]{4}{*}{$<0.001$} & $30.0(28.5-31.5)$ & $31.9(30.2-33.6)$ & \multirow[t]{4}{*}{$<0.001$} \\
\hline Primary & 29.2 & $28.5(27.6-29.3)$ & $29.9(28.8-31.0)$ & & $28.4(27.1-29.7)$ & $32.3(30.6-33.9)$ & \\
\hline Secondary & 34.0 & $33.6(32.6-34.7)$ & $34.4(33.1-35.7)$ & & $35.1(33.5-36.6)$ & $31.3(29.7-33.0)$ & \\
\hline Higher & 7.7 & $7.3(6.8-7.8)$ & $5.4(4.9-5.9)$ & & $6.6(5.9-7.3)$ & $4.6(3.9-5.3)$ & \\
\hline \multicolumn{8}{|l|}{ Religion } \\
\hline Islam & 90.0 & 90.4 (89.4-91.3) & $92.0(91.0-93.0)$ & \multirow[t]{2}{*}{0.021} & $91.4(90.2-92.4)$ & $91.0(89.6-92.2)$ & \multirow[t]{2}{*}{0.014} \\
\hline Other & 10.0 & $9.6(8.7-10.6)$ & $8.0(7.0-9.0)$ & & $8.6(7.6-9.8)$ & $9.0(7.8-10.4)$ & \\
\hline
\end{tabular}


Table A1. Cont.

\begin{tabular}{|c|c|c|c|c|c|c|c|}
\hline & & \multicolumn{2}{|c|}{ Fever $(N=30,731)$} & \multirow[t]{2}{*}{$p$-Value } & \multicolumn{2}{|c|}{ Dyspnoea $(N=11,354)$} & \multirow[t]{2}{*}{$p$-Value } \\
\hline & & No & Yes & & No & Yes & \\
\hline \multicolumn{8}{|l|}{ Sanitation } \\
\hline Unimproved & 45.6 & $47.9(46.6-49.2)$ & $49.3(47.7-50.8)$ & & $47.8(46.0-49.6)$ & $51.6(49.6-53.6)$ & \\
\hline Improved & 54.4 & $52.1(50.8-53.4)$ & $50.7(49.2-52.3)$ & & $52.2(50.4-54.0)$ & $48.4(46.4-50.4)$ & \\
\hline \multicolumn{8}{|l|}{ Water } \\
\hline Unimproved & 6.8 & $7.0(6.5-7.6)$ & $6.4(5.7-7.1)$ & $<0.001$ & $6.3(5.5-7.1)$ & $6.6(5.5-7.3)$ & $<0.001$ \\
\hline Improved & 93.2 & $93.0(92.4-93.5)$ & $93.6(92.9-94.3)$ & & $93.7(92.9-94.5)$ & $93.4(92.2-95.8)$ & \\
\hline \multicolumn{8}{|l|}{ Cooking Fuel } \\
\hline Unclean & 89.0 & $88.0(86.6-89.2)$ & $90.9(89.6-92.0)$ & & $88.8(87.1-90.3)$ & $92.5(91.1-93.6)$ & \\
\hline Clean & 11.0 & $12.0(10.8-13.4)$ & $9.1(8.0-10.4)$ & & $11.2(9.7-12.9)$ & $7.5(6.4-8.9)$ & \\
\hline \multicolumn{8}{|c|}{ Wealth Quintile } \\
\hline Poorest & 20.0 & $20.3(19.3-21.4)$ & $23.1(21.9-24.4)$ & $<0.001$ & $20.9(19.6-22.3)$ & $25.7(24.0-27.5)$ & $<0.001$ \\
\hline Poorer & 20.0 & $20.5(19.7-21.3)$ & $20.6(19.6-21.7)$ & & $19.6(18.4-20.9)$ & $22.1(20.6-23.6)$ & \\
\hline Middle & 18.8 & $18.5(17.8-19.3)$ & $19.8(18.6-21.0)$ & & $19.1(17.8-20.5)$ & $19.3(18.0-20.7)$ & \\
\hline Richer & 19.4 & $19.6(18.8-20.5)$ & $19.0(18.0-20.0)$ & & $19.7(18.5-21.0)$ & $17.5(16.2-18.9)$ & \\
\hline Richest & 21.8 & $21.1(19.9-22.2)$ & $17.5(16.3-18.7)$ & & $20.7(19.2-22.3)$ & $15.4(14.2-16.8)$ & \\
\hline \multicolumn{8}{|l|}{ Employment } \\
\hline Unemployed & 75.8 & $75.3(74.0-76.6)$ & $75.1(73.6-76.6)$ & 0.021 & $74.8(73.0-76.6)$ & $76.9(74.9-78.8)$ & 0.017 \\
\hline Employed & 24.2 & $24.7(23.4-26.0)$ & $24.9(23.4-26.4)$ & & $25.2(23.4-27.0)$ & $23.1(21.2-25.1)$ & \\
\hline \multicolumn{8}{|l|}{ Parity } \\
\hline 2 & 59.0 & $59.4(58.4-60.4)$ & $58.1(57.0-59.3)$ & 0.041 & $59.4(57.9-60.9)$ & $57.9(56.3-59.5)$ & 0.018 \\
\hline 4 & 27.6 & $27.1(26.4-27.9)$ & $28.5(27.5-29.4)$ & & $28.4(27.2-29.7)$ & $27.6(26.3-29.0)$ & \\
\hline 5 & 13.3 & $13.5(12.8-14.2)$ & $13.4(12.6-14.3)$ & & $12.2(11.2-13.2)$ & $14.5(13.3-15.8)$ & \\
\hline \multicolumn{8}{|l|}{ Age of Child } \\
\hline $0-11$ & 24.3 & $23.0(22.3-23.7)$ & $27.3(26.3-28.4)$ & $<0.001$ & $25.6(24.4-26.9)$ & $32.0(30.4-33.7)$ & 0.024 \\
\hline $12-23$ & 21.0 & $20.3(19.5-21.1)$ & $23.4(22.4-24.4)$ & & $22.4(21.1-23.7)$ & $23.5(22.0-25.0)$ & \\
\hline $24-35$ & 20.9 & $20.5(19.8-21.2)$ & $19.7(18.9-20.6)$ & & $20.3(19.1-21.5)$ & $18.7(17.5-20.0)$ & \\
\hline $36-47$ & 18.9 & $19.8(19.1-20.5)$ & $16.5(15.6-17.4)$ & & $17.7(16.6-18.9)$ & $14.4(13.2-15.6)$ & \\
\hline $48-59$ & 14.9 & $16.5(15.8-17.2)$ & $13.0(12.0-14.0)$ & & $14.0(12.7-15.4)$ & $11.4(10.4-12.6)$ & \\
\hline \multicolumn{8}{|l|}{ Child's Sex } \\
\hline Male & 50.9 & $51.1(50.3-52.0)$ & $51.6(50.5-52.6)$ & 0.128 & $51.3(49.9-52.7)$ & $52.7(51.0-54.3)$ & 0.191 \\
\hline Female & 49.1 & $48.9(48.0-49.7)$ & $48.4(47.4-49.5)$ & & $48.7(47.3-50.1)$ & $47.3(45.7-49.0)$ & \\
\hline
\end{tabular}

Note: confidence intervals shown in parentheses. 
Table A2. Multicollinearity tests.

\begin{tabular}{|c|c|}
\hline \multicolumn{2}{|c|}{ Multicollinearity Test for Outcome 1 (Fever): } \\
\hline Variable & VIF \\
\hline Water & 8.91 \\
\hline Parity & 8.23 \\
\hline Age & 7.66 \\
\hline Residency & 7.44 \\
\hline Wealth & 6.96 \\
\hline Child's Sex & 5.69 \\
\hline Region & 4.77 \\
\hline Education & 4.43 \\
\hline Sanitation & 3.80 \\
\hline Fuel & 1.55 \\
\hline Occupation & 1.34 \\
\hline Religion & 1.13 \\
\hline \multicolumn{2}{|c|}{ Multicollinearity test for outcome 2 (dyspnoea) } \\
\hline Variable & VIF \\
\hline Water & 8.46 \\
\hline Parity & 7.16 \\
\hline Age & 5.31 \\
\hline Residency & 5.14 \\
\hline Wealth & 4.96 \\
\hline Child's Sex & 4.12 \\
\hline Region & 3.17 \\
\hline Education & 3.23 \\
\hline Sanitation & 2.17 \\
\hline Fuel & 1.55 \\
\hline Occupation & 1.32 \\
\hline Religion & 1.13 \\
\hline
\end{tabular}

\section{References}

1. WB: Bangladesh Has 8th Highest Number of Poor People. Available online: https://www. dhakatribune.com/business/2016/10/04/wb-bangladesh-8th-highest-number-poor-people (accessed on 1 December 2018).

2. Top 10 Disturbing Facts about Poverty in Bangladesh. Available online: https://borgenproject.org/10-factsabout-poverty-in-bangladesh/ (accessed on 1 December 2018).

3. Koblinsky, M.; Anwar, I.; Mridha, M.K.; Chowdhury, M.E.; Botlero, R. Reducing Maternal Mortality and Improving Maternal Health: Bangladesh and MDG 5. J. Health Popul. Nutr. 2008, 26, 280-294. [CrossRef] [PubMed]

4. Jolly, S.P.; Rahman, M.; Afsana, K.; Yunus, F.M.; Chowdhury, A.M.R. Evaluation of Maternal Health Service Indicators in Urban Slum of Bangladesh. PLoS ONE 2016, 11, e0162825. [CrossRef] [PubMed]

5. Ruhul, A.M.; Fukuda, H.; Nakajima, K.; Takatorige, T.; Tatara, K. Public health services in Bangladesh with special reference to systems and trends of vital statistics. Environ. Health Prev. Med. 1999, 4, 65-70. [CrossRef] [PubMed] 
6. Cockcroft, A.; Milne, D.; Oelofsen, M.; Karim, E.; Andersson, N. Health services reform in Bangladesh: Hearing the views of health workers and their professional bodies. BMC Health Serv. Res. 2011, 11, S8. [CrossRef] [PubMed]

7. Ahmed, S.; Hasan, M.Z.; Ahmed, M.W.; Dorin, F.; Sultana, M.; Islam, Z.; Mirelman, A.J.; Rehnberg, C.; Khan, J.A.M.; Chowdhury, M.E. Evaluating the implementation related challenges of Shasthyo Suroksha Karmasuchi (health protection scheme) of the government of Bangladesh: A study protocol. BMC Health Serv. Res. 2018, 18, 552. [CrossRef] [PubMed]

8. Molla, A.A.; Chi, C. Who pays for healthcare in Bangladesh? An analysis of progressivity in health systems financing. Int. J. Equity Health 2017, 16, 167. [CrossRef] [PubMed]

9. 33 Million Children in Bangladesh Live in Poverty. Available online: https://www.unicef.org/media/ media_51925.html (accessed on 1 December 2018).

10. Bouzid, M.; Cumming, O.; Hunter, P.R. What is the impact of water sanitation and hygiene in healthcare facilities on care seeking behaviour and patient satisfaction? A systematic review of the evidence from low-income and middle-income countries. BMJ Glob. Health 2018, 3, e000648. [CrossRef]

11. Chard, A.N.; Trinies, V.; Moss, D.M.; Chang, H.H.; Doumbia, S.; Lammie, P.J.; Freeman, M.C. The impact of school water, sanitation, and hygiene improvements on infectious disease using serum antibody detection. PLoS Negl. Trop. Dis. 2018, 12, e0006418. [CrossRef]

12. Dreibelbis, R.; Freeman, M.C.; Greene, L.E.; Saboori, S.; Rheingans, R. The Impact of School Water, Sanitation, and Hygiene Interventions on the Health of Younger Siblings of Pupils: A Cluster-Randomized Trial in Kenya. Am. J. Public Health 2014, 104, e91-e97. [CrossRef]

13. Hutton, G. Global costs and benefits of reaching universal coverage of sanitation and drinking-water supply. J. Water Health 2013, 11, 1-12. [CrossRef]

14. Water in Crisis-Spotlight Nepal. Available online: https://thewaterproject.org/water-crisis/water-incrisis-nepal (accessed on 20 February 2018).

15. Rahman, M.; Ashraf, S.; Unicomb, L.; Mainuddin, A.K.M.; Parvez, S.M.; Begum, F.; Das, K.K.; Naser, A.M.; Hussain, F.; Clasen, T.; et al. WASH Benefits Bangladesh trial: System for monitoring coverage and quality in an efficacy trial. Trials 2018, 19,360. [CrossRef] [PubMed]

16. Lin, A.; Ercumen, A.; Benjamin-Chung, J.; Arnold, B.F.; Das, S.; Haque, R.; Ashraf, S.; Parvez, S.M.; Unicomb, L.; Rahman, M.; et al. Effects of Water, Sanitation, Handwashing, and Nutritional Interventions on Child Enteric Protozoan Infections in Rural Bangladesh: A Cluster-Randomized Controlled Trial. Clin. Infect. Dis. 2018, 67, 1515-1522. [CrossRef] [PubMed]

17. Raihan, M.J.; Farzana, F.D.; Sultana, S.; Haque, M.A.; Rahman, A.S.; Waid, J.L.; McCormick, B.; Choudhury, N.; Ahmed, T. Examining the relationship between socio-economic status, WASH practices and wasting. PLoS ONE 2017, 12, e0172134. [CrossRef] [PubMed]

18. WHO, Bangladesh-Sustainable Development \& Healthy Environment. Available online: http://www. whoban.org/sust_dev_mental_env.html (accessed on 1 December 2018).

19. Simoes, E.A.F.; Cherian, T.; Chow, J.; Shahid-Salles, S.A.; Laxminarayan, R.; John, T.J. Acute Respiratory Infections in Children. In Disease Control Priorities in Developing Countries; Jamison, D.T., Breman, J.G., Measham, A.R., Alleyne, G., Claeson, M., Evans, D.B., Jha, P., Mills, A., Musgrove, P., Eds.; World Bank: Washington, DC, USA, 2006; ISBN 978-0-8213-6179-5.

20. Liu, W.K.; Liu, Q.; Chen, D.H.; Liang, H.X.; Chen, X.K.; Chen, M.X.; Qiu, S.Y.; Yang, Z.Y.; Zhou, R. Epidemiology of Acute Respiratory Infections in Children in Guangzhou: A Three-Year Study. PLoS ONE 2014, 9, e96674. [CrossRef] [PubMed]

21. Cutrera, R.; Baraldi, E.; Indinnimeo, L.; Miraglia Del Giudice, M.; Piacentini, G.; Scaglione, F.; Ullmann, N.; Moschino, L.; Galdo, F.; Duse, M. Management of acute respiratory diseases in the pediatric population: The role of oral corticosteroids. Ital. J. Pediatr. 2017, 43, 31. [CrossRef] [PubMed]

22. Harerimana, J.-M.; Nyirazinyoye, L.; Thomson, D.R.; Ntaganira, J. Social, economic and environmental risk factors for acute lower respiratory infections among children under five years of age in Rwanda. Arch. Public Health 2016, 74, 19. [CrossRef] [PubMed]

23. Selvaraj, K.; Chinnakali, P.; Majumdar, A.; Krishnan, I.S. Acute respiratory infections among under-5 children in India: A situational analysis. J. Nat. Sci. Biol. Med. 2014, 5, 15-20. [CrossRef] [PubMed]

24. Nandasena, S.; Wickremasinghe, A.R.; Sathiakumar, N. Indoor air pollution and respiratory health of children in the developing world. World J. Clin. Pediatr. 2013, 2, 6-15. [CrossRef] 
25. Akinyemi, J.O.; Morakinyo, O.M. Household environment and symptoms of childhood acute respiratory tract infections in Nigeria, 2003-2013: A decade of progress and stagnation. BMC Infect. Dis. 2018, 18, 296. [CrossRef]

26. Humans, I.W.G. on the E. of C.R. Exposure Data; International Agency for Research on Cancer: Lyon, France, 2010.

27. Salje, H.; Gurley, E.S.; Homaira, N.; Ram, P.K.; Haque, R.; Petri, W.; Moss, W.J.; Luby, S.P.; Breysse, P.; Azziz-Baumgartner, E. Impact of neighborhood biomass cooking patterns on episodic high indoor particulate matter concentrations in clean fuel homes in Dhaka, Bangladesh. Indoor Air 2014, 24, 213-220. [CrossRef]

28. Tanchangya, J.; Geater, A.F. Use of traditional cooking fuels and the risk of young adult cataract in rural Bangladesh: A hospital-based case-control study. BMC Ophthalmol. 2011, 11, 16. [CrossRef] [PubMed]

29. North, C.M.; Valeri, L.; Hunt, P.W.; Mocello, A.R.; Martin, J.N.; Boum, Y.; Haberer, J.E.; Bangsberg, D.R.; Christiani, D.C.; Siedner, M.J. Cooking fuel and respiratory symptoms among people living with HIV in rural Uganda. ERJ Open Res. 2017, 3, 00094-2016. [CrossRef] [PubMed]

30. Kilabuko, J.H.; Nakai, S. Effects of Cooking Fuels on Acute Respiratory Infections in Children in Tanzania. Int. J. Environ. Res. Public Health 2007, 4, 283-288. [CrossRef] [PubMed]

31. Ghose, B.; Feng, D.; Tang, S.; Yaya, S.; He, Z.; Udenigwe, O.; Ghosh, S.; Feng, Z. Women's decision-making autonomy and utilisation of maternal healthcare services: Results from the Bangladesh Demographic and Health Survey. BMJ Open 2017, 7, e017142. [CrossRef] [PubMed]

32. National Institute of Population Research and Training, Mitra and Associates; ORC Macro. Bangladesh Demographic and Health Survey 2014; ORC Macro: Dhaka, Bangladesh, 2014.

33. Nasreen, S.; Luby, S.P.; Brooks, W.A.; Homaira, N.; Mamun, A.A.; Bhuiyan, M.U.; Rahman, M.; Ahmed, D.; Abedin, J.; Rahman, M.; et al. Population-Based Incidence of Severe Acute Respiratory Virus Infections among Children Aged <5 Years in Rural Bangladesh, June-October 2010. PLoS ONE 2014, 9, e89978. [CrossRef] [PubMed]

34. Chowdhury, A.H.; Hanifi, S.M.A.; Mia, M.N.; Bhuiya, A. Socioeconomic inequalities in under-five mortality in rural Bangladesh: Evidence from seven national surveys spreading over 20 years. Int. J. Equity Health 2017, 16, 197. [CrossRef] [PubMed]

35. Bishwajit, G. Household wealth status and overweight and obesity among adult women in Bangladesh and Nepal. Obes. Sci. Pract. 2017, 3, 185-192. [CrossRef] [PubMed]

36. Giashuddin, S.M.; Rahman, A.; Rahman, F.; Mashreky, S.R.; Chowdhury, S.M.; Linnan, M.; Shafinaz, S. Socioeconomic inequality in child injury in Bangladesh-Implication for developing countries. Int. J. Equity Health 2009, 8, 7. [CrossRef] [PubMed]

37. Rahman, M.M.; Shahidullah, M. Risk factors for acute respiratory infections among the slum infants of Dhaka city. Bangladesh Med. Res. Counc. Bull. 2001, 27, 55-62. [PubMed]

38. Kumar, S.G.; Majumdar, A.; Kumar, V.; Naik, B.N.; Selvaraj, K.; Balajee, K. Prevalence of acute respiratory infection among under-five children in urban and rural areas of puducherry, India. J. Nat. Sci. Biol. Med. 2015, 6, 3-6. [CrossRef]

39. Holloway, K.A.; Karkee, S.B.; Tamang, A.; Gurung, Y.B.; Kafle, K.K.; Pradhan, R.; Reeves, B.C. Community intervention to promote rational treatment of acute respiratory infection in rural Nepal. Trop. Med. Int. Health 2009, 14, 101-110. [CrossRef] [PubMed]

40. Khan, T.A.; Madni, S.A.; Zaidi, A.K.M. Acute respiratory infections in Pakistan: Have we made any progress? J. Coll. Phys. Surg. Pak. 2004, 14, 440-448.

41. Chowdhury, F.; Sturm-Ramirez, K.; Mamun, A.A.; Iuliano, A.D.; Bhuiyan, M.U.; Chisti, M.J.; Ahmed, M.; Haider, S.; Rahman, M.; Azziz-Baumgartner, E. Factors driving customers to seek health care from pharmacies for acute respiratory illness and treatment recommendations from drug sellers in Dhaka city, Bangladesh. Patient Prefer. Adher. 2017, 11, 479-486. [CrossRef] [PubMed]

42. Halder, A.K.; Luby, S.P.; Akhter, S.; Ghosh, P.K.; Johnston, R.B.; Unicomb, L. Incidences and Costs of Illness for Diarrhea and Acute Respiratory Infections for Children <5 Years of Age in Rural Bangladesh. Am. J. Trop. Med. Hyg. 2017, 96, 953-960. [PubMed]

43. Gupta, R.P.-S.; de Wit, M.L.; McKeown, D. The impact of poverty on the current and future health status of children. Paediatr. Child Health 2007, 12, 667-672. [CrossRef] [PubMed] 
44. Yaya, S.; Hudani, A.; Udenigwe, O.; Shah, V.; Ekholuenetale, M.; Bishwajit, G. Improving Water, Sanitation and Hygiene Practices, and Housing Quality to Prevent Diarrhea among Under-Five Children in Nigeria. Trop. Med. Infect. Dis. 2018, 3, 41. [CrossRef] [PubMed]

45. Onozuka, D.; Chaves, L.F. Climate Variability and Nonstationary Dynamics of Mycoplasma pneumoniae Pneumonia in Japan. PLoS ONE 2014, 9, e95447. [CrossRef] [PubMed] 